\title{
Re: Treatment of Peritoneal Carcinomatosis by Cytoreductive Surgery and Intraperitoneal Hyperthermic Chemoperfusion (IHCP): Postoperative Outcome and Risk Factors for Morbidity
}

eritoneal carcinomatosis results from intraperitoneal dissemination of cells derived of primary tumours from various origin. The treatment of this tumour stage is generally palliative. Beside ovarian cancer, peritoneal carcinomatosis from other origin does little react upon systemic chemotherapy only. Therefore, more demanding therapies are being explored. To date, one of the most successful therapeutic concepts consists of radical surgical tumor debulking followed by intraoperatively applied intraperitoneal hyperthermic chemotherapy.

Currently several components of this therapy are under investigation to optimize effect and to reduce side effects. Obviously the more complete the reduction of tumor tissue can be achieved, the higher the chemoconcentration in residual tumour tissue will be. In general, a cytoreduction to no macroscopic detectable disease or at least a reduction to tumour nodules of $<2.5 \mathrm{~mm}$ of diameter was the goal. ${ }^{1}$ For the intraoperative intraperitoneal chemotherapy the abdominal cavity can be temporarily closed or left open. The latter has the advantage that local hyperthermia and chemoperfusion within the peritoneal cavity will be more homogeneous. ${ }^{2}$

Based on the experience with the treatment of the pseudomyxoma peritoneii, using Mitomycin $\mathrm{C}$ in combination with 5-FU as chemotherapeutic agents. However, the effect on colorectal cancer is limited and recently Oxaliplatin and Irinotecan have been used for intraperitoneal therapy in these diseases.,4 Furthermore, for mesotheliomas and ovarian cancer related peritoneal carcinomatosis, Paclitaxel can be used successfully, as local peritoneal tissue concentrations exceeds $10^{3}$ times compared to the concentrations achieved with intravenous application of the drug. ${ }^{5}$

In the present issue, Roviello et al. ${ }^{6}$ (DOI: $10.1007 /$ s00268-006-0038-0) report their experience $(N=61)$ with cytoreduction and intraperitoneal hyperthermic chemoperfusion and present their outcome and morbidity. In their series, the authors confirm that the median survival is potentially by far better after this complex therapeutic strategy, than following systemic chemotherapy only. However, if this is the effect of aggressive cytoreduction or if it can be attributed to the intraperitoneal, chemotherapy is still open.

As expected and confirmed by this study, age (RR 1.06 per year) is a significant risk factor for complications following the presented therapy. ${ }^{6}$ One would expect extent of disease, visceral anastomosis or extent of resection (peritonectomy, time of duration of the procedure) as additional significant risk factors for morbidity. However completeness of cancer resection (CCR 2/3 versus CCR 0/1: RR 9.27) showed to have highest impact upon complication frequency in the present patient series. ${ }^{6}$ In cases where complete cytoreduction could to be achieved (CCR 2/3) the fact of high morbidity may reflect the difficulty or "risk taking" of the surgical procedure to reduce tumour tissue to the desired extent in the individual patient.

In summary, Roviello et al. ${ }^{6}$ have highlighted the advantage of surgical cytoreduction in combination with intraperitoneal hyperthermic chemotherapy in respect of survival as well as the risk factors related to complications. However, the treatment of peritoneal carcinoma- 
tosis is demanding, associated with substantial morbidity and is still under investigation for further improvements and therefore should only be done in tertiary referral centres preferentially within study protocols.

\section{REFERENCES}

1. Sugarbaker PH. Cytoreductive surgery and peri-operative intraperitoneal chemotherapy as a curative approach to pseudomyxoma peritonei syndrome. Eur J Surg Oncol 2001;27:239-43.

2. Sugarbaker PH, Chang D. Results of treatment of 385 patients with peritoneal surface spread of appendiceal malignancy. Ann Surg Oncol 1999;6:727-731.

3. Elias D, Matsuhisa T, Sideris $L$, et al. Heated intra-operative intraperitoneal exaliplatin plus irinotecan after complete resection of peritoneal carcinomatosis: pharmacokinetics, tissue distribution and tolerance. Ann Oncol 2004;15:15581565.
4. Elias D, Sideris L, Pocard M, et al. Efficacy of intraperitoneal chemohyperthermia with oxaliplatin in colorectal peritoneal carcinomatosis. Preliminary results in 24 patients. Ann Oncol 2004;15:781-785.

5. Markmann M, Rowinsky E, Hakes T, et al. Phase I trial of intraperitoneal taxol: a Gynecologic Oncology Group study. $\mathrm{J}$ Clin Oncol 1992;10:1485-1491.

6. Roviello F, Marrelli D, Neri A, et al. Treatment of Peritoneal Carcinomatosis by Cytoreductive Surgery and Intraperitoneal Hyperthermic Chemoperfusion (IHCP): Postoperative Outcome and Risk Factors for Morbidity World J Surg 2006; 30 (DOI: 10.1007/s00268-006-0038-0).

\section{Walter Richard Marti, MD, FACS}

Department of Visceral Surgery,

University of Basel, Spitalstrasse 21, CH, 4031 Basel, Switzerland

e-mail:wrmarti@uhbs.ch 